\title{
|?
}

\section{PATENTALAVA. DINÁMICA DE LAS ESTRATEGIAS DE INNOVACIÓNY SU RELACIÓN CON LA EVOLUCIÓN DE LAS PATENTES. EL CASO ALAVÉS*}

\author{
ROSA MARÍA RÍO \\ JESÚS MARÍA LARRAÑAGA \\ FERNANDO ELIZAGARATE \\ DEPARTAMENTO DE ORGANIZACIÓN \\ ESCUELA UNIVERSITARIA DE INGENIERÍA DE VITORIA-GASTEIZ \\ UNIVERSIDAD DEL PAÍS VASCO
}

\begin{abstract}
Resumen: La información contenida en bases de datos de patentes puede ser usada para visualizar la estrategia de innovación de una región. El trabajo denominado PATENTALAVA se basa en el uso de la patentometria, técnica en estado embrionario, para estudiar la evolución de los flujos de conocimiento interindustrial. Este conocimiento permite el establecimiento de políticas eficaces que mejoren la competitividad industrial de la región. En Patentalava se analizan las estadísticas de los registros de patentes y modelos de utilidad solicitados por empresas de la zona, a continuación se visualiza la evolución innovadora por sectores empresariales y se llega a representar mediante análisis Cluster las áreas emergentes de patentabilidad así como sus relaciones. La detección de los transvases de conocimiento desde sectores tradicionales a sectores emergentes permite establecer conclusiones vitales cuando se trata de definir la estrategia de innovación en una región.
\end{abstract}

Palabras clave: Patent analysis, patent mining, innovation, knowledge management.

\section{Objetivo y alcance}

En este trabajo se analiza la evolución del progreso tecnológico en Álava a través del estudio de las pa- tentes solicitadas desde dicha región española. Se relacionan los solicitantes y su localización con la Clasificación Internacional de sus inventos mostrando las áreas innovadoras además se grafican los movimientos y flujos de conocimiento industrial de la región alavesa a través de la evolución de la innovación en los polígonos industriales. Por último, se localizan los polos tecnológicos emergentes resultado de las estrategias de innovación en los sectores industriales. En definitiva PatentAlava analiza la evolución de la innovación en Alava a través de un análisis histórico del Registro de la Propiedad Industrial.

\section{Estado del arte}

El uso de las patentes para evaluar la innovación en un país determinado ha sido abordado por diversos autores como Tansey y Stembridge (2005). El tópico de las patentes como fuentes de conocimiento tecnológico se puede encontrar en Gray and Meister (2006) y los métodos de extracción de información han sido expuestos por Zhu, and Porter (2002) y otros autores. Sin embargo pocas son las referencias a la utilización del análisis de patentes en sectores concretos y menos aún la denominación de Paten-

\footnotetext{
* Este trabajo es consecuencia de la participación de los autores en un proyecto de investigación financiado por la UPV/EHU con referencia NUPV06/20, titulado «Patentalava. Dinámica de las estrategias de innovación y la evolución del Registro de la Propiedad Industrial. El caso alavés».
} 
tometría para realizar estudios como el titulado Benchmarking R\&D elaborado por Dou (2004). En el presente trabajo se toman como referencia estudios como el de Han et al. (2006) que intentan determinar el conocimiento inter-industrial a través del análisis de patentes o la herramienta Patent portfolio análisis que realiza Fabry et al. (2006) al objeto de identificar I+D y señalar oportunidades de negocio.

\section{Muestra}

Se obtuvieron de la base de datos de invenciones en español de la Oficina Española de Patentes y Marcas los Registros de Patentes y Modelos de Utilidad solicitadas por particulares o empresas alavesas desde el 25 de mayo de 1960 hasta el 3 I de diciembre de 2006 En la Figura I se muestra un registro tipo analizado.

La Muestra se compone de 2.297 patentes de las cuales solo hay un $8 \%$ no concedidas o rechazadas. Por tanto, las patentes solicitadas son bastante robustas, lo que refleja que cuando se llega al Registro Industrial la innovación conseguida es exitosa y novedad mundial. A fecha de abril 2007 se encuentran vigentes el $63 \%$ de las concedidas. De las patentes vigentes el $55 \%$ le quedan menos de diez años de vigencia y al $45 \%$ más de diez años. En resumen al 29\% de las patentes solicitadas y concedidas le quedan más de diez años de vida, este dato enmarcará las posteriores conclusiones sobre el avance de la I+D en Álava. La evolución de las solicitudes muestra un descenso en las mismas continuo a lo largo de las décadas a razón del 3,7\% anual.

\section{Análisis realizados}

Una vez filtrada, se preparo la información para ser importada por los programas SPSS 14.0 y Vantage point (software bibliométrico) y posteriormente analizada utilizando diferentes técnicas: recuentos estadísticos, frecuencias, agrupamientos, co-ocurrencia de palabras, análisis cluster. Presentamos en este apartado cuarto algunos de los resultados de esta investigación.

\section{I. Indicadores de primera generación relevantes}

\subsubsection{Análisis de solicitantes}

El $58 \%$ de las patentes y modelos de utilidad han sido solicitadas a nombre de particulares aunque,
Figura

Registro Tipo

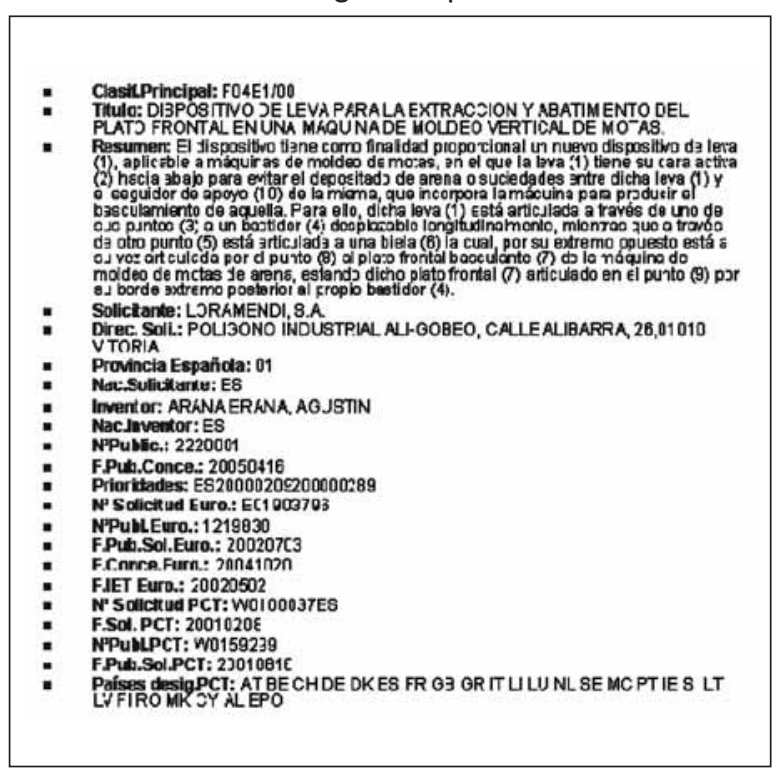

sin embargo el $42 \%$ ha sido solicitado por empresas. La nacionalidad de los solicitantes es en un 97,34\% española. Las diez empresas alavesas que más patentes han solicitado se pueden apreciar en la Tabla I.

\subsubsection{Patentes, modelos de utilidad y actividades industriales}

Podemos analizar la evolución de los polígonos industriales en Alava a través de las Patentes solicitadas por las empresas en ellos localizadas. Así, podremos ver evolucionar los polígonos desde la década de los años 60 hasta el año 2006 y comparar la estructura y sectores implantados en los mismos. Por ejemplo se puede apreciar la influencia del Parque tecnológico que en la década de los años 90 se posiciona con un $1 \%$ de las innovaciones solicitadas y en los años 2000-2006 ya alcanza el 17\% de las innovaciones solicitadas en la provincia. Los resultados se muestran en la Figura 3.

Si agrupamos las solicitudes en base a la correspondencia de sectores con los códigos de la Clasificación Internacional de patentes dada por Hidalgo (2003) podemos determinar que los sectores industriales que han movilizado la I+D en Álava han sido con un 37,28\% la ingeniería civil y los bienes de consumo, en un $25,72 \%$ la ingeniería mecánica y maquinaria, el un 18,93\% la ingeniería de proceso, el 8,67\% la electricidad y electrónica el 8, I 4\% la instrumentación, y el I,26\% la Química-Farmacia. Se muestra en la Figura 4 la evolución de la innovación, 
Figura 2

Evolución de las solicitudes de patentes y modelos de utilidad (1978-2006)

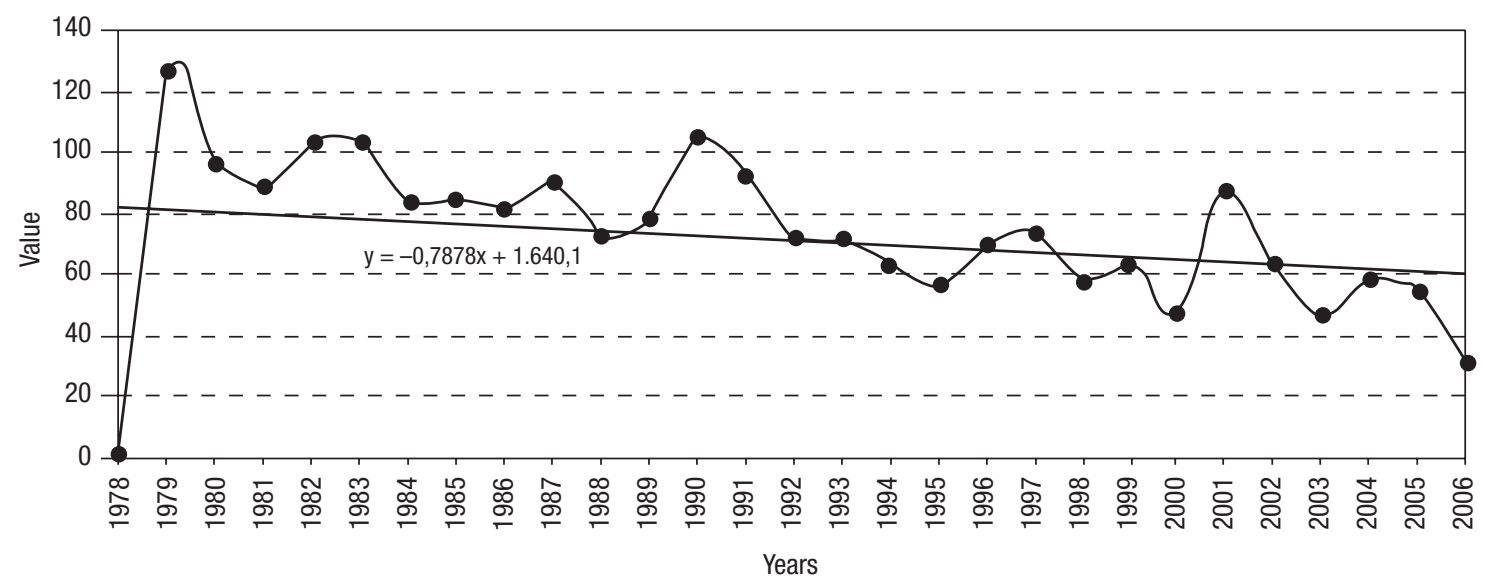

medida como patentabilidad, en los sectores industriales a lo largo de los años (1960-2006). Se aprecia la tendencia decreciente.

\subsection{Análisis cluster. Ejemplo}

Mediante diversos cruzamientos entre los campos de los registros podemos llegar a graficar la información y extraer interesantes conclusiones. El primero de los cruzamientos relaciona la Clasificación Internacional de Patentes con el campo Solicitante, lo que nos permite visualizar las relaciones entre las empresas innovadoras así como la calidad o fuerza de esas relaciones. Por otra parte si estudiamos la Clasificación Internacional en relación a la fecha de solicitud podremos determinar como se están configurando las nuevas áreas de innovación del territorio.

\subsubsection{Cluster tecnológicos o el Panorama Industrial Innovador alavés}

Analizamos los clusters tecnológicos innovadores a través del estudio de la Clasificación Internacional de

Tabla I

Top ten solicitantes alaveses

\begin{tabular}{|c|c|l|l|}
\hline & N. ${ }^{\circ}$ patentes & \multicolumn{1}{|c|}{ Denominación del solicitante } & \multicolumn{1}{|c|}{ Actividad } \\
\hline 1 & 108 & INOVAC-RIMA, S. A. & Juguetera \\
\hline 2 & 71 & LORAMENDI Coop. & Fundición \\
\hline 3 & 58 & Celaya Emparanza y Galdos, S. A. CEGASA & Pilas portátiles \\
\hline 4 & 40 & LIPMESA. La Indusrial Plástica y Metalúrgica, S.A. & Amortiguadores, resortes neumáticos \\
\hline 5 & 38 & TERRAIN Ibérica, S. A., Y Terrain SDP, S. A. & Tuberías y accesorios PVC \\
\hline 6 & 35 & MCM Metalúrgica Cerrajera Mondragón, S. A. & $\begin{array}{l}\text { Fabricación de cerraduras para } \\
\text { contrucción }\end{array}$ \\
\hline 7 & 33 & SAGOLA, S.A. & Herramientas y equipos de pulverización \\
\hline 8 & 32 & $\begin{array}{l}\text { RODOLFO BACAICOA CASTELLANOS } \\
\text { (ZIQUESE, S.L.) }\end{array}$ & $\begin{array}{l}\text { Mejoras para máquinas recreativas y } \\
\text { mesas de billar }\end{array}$ \\
\hline 9 & 30 & DAIXALUX, S.A. & Alumbrado de emergencia \\
\hline 10 & 29 & ANA URGUIZU OSA (EGA MASTER, S.A.) & Mejoras en herramientas manuales \\
\hline
\end{tabular}


Figura 3

Evolución de la innovación en los Polígonos Industriales Alaveses

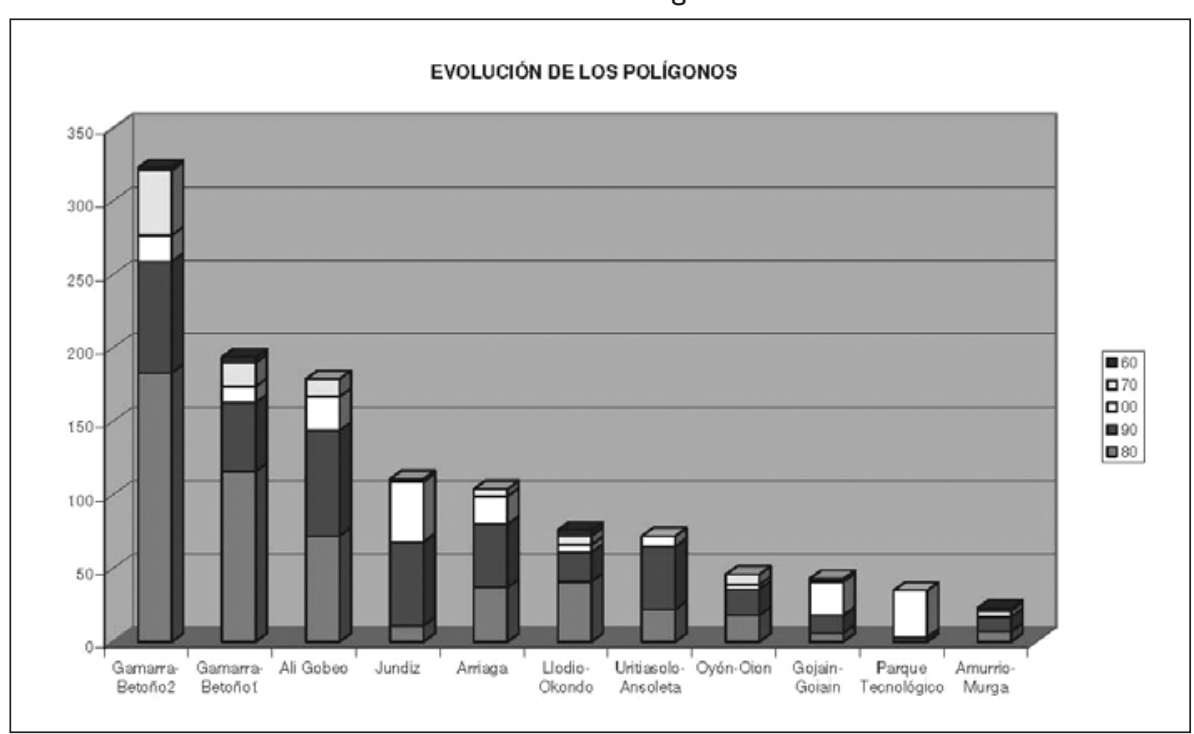

las patentes solicitadas en cada uno de los 2297 registros; así, podremos graficar el Panorama Industrial Innovador alaves (Fig. 5) Para la elaboración del grafico se han tenido en cuenta los solicitantes con diez o más patentes. En él se aprecia la existencia de cinco clusters bien definidos:

- CLUSTER I: Inovac-Rima S.A., Ana Urquizu Osa ( Ega Master S.A.), BSH Krainel Comercial UNESA S.A., BSH Comercial, Kemen, Ofita y Home Fittiting España S.A... y otros. Todas ellas tienen en común patentar en el sector de bienes de consumo y equipos.
- Cluster 2: Cevi S.L., Rodolfo Bacaicoa CasteIlanos (ZIQUESA S.L.), Euroenvases S.A.... y otros. El sector en el cual han basado su actividad innovadora estas empresas es el de tecnologías de control, análisis y medida. Sin embargo también cuentan con patentes en el sector de los bienes de consumo y equipos de ahí su proximidad con el cluster I en el diagrama.

- ClUSTER 3: Claramente diferenciado, compuesto por empresas como Poza S.A. y Gamesa Desarrollos Aeronáuticos S.A. Estas empresas han destacado por innovar en el sector del transporte.

Figura 4

Evolución de los Sectores Industriales

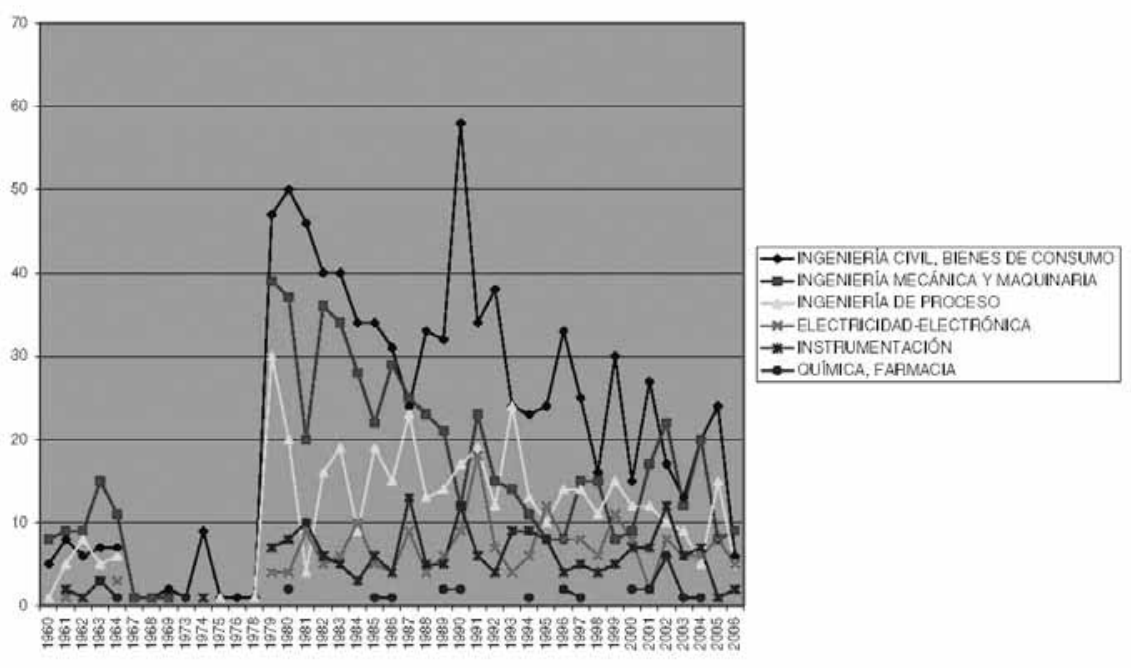


Figura 5

Clusters tecnológicos en el Panorama Industrial Alavés (Solicitante-CIP)

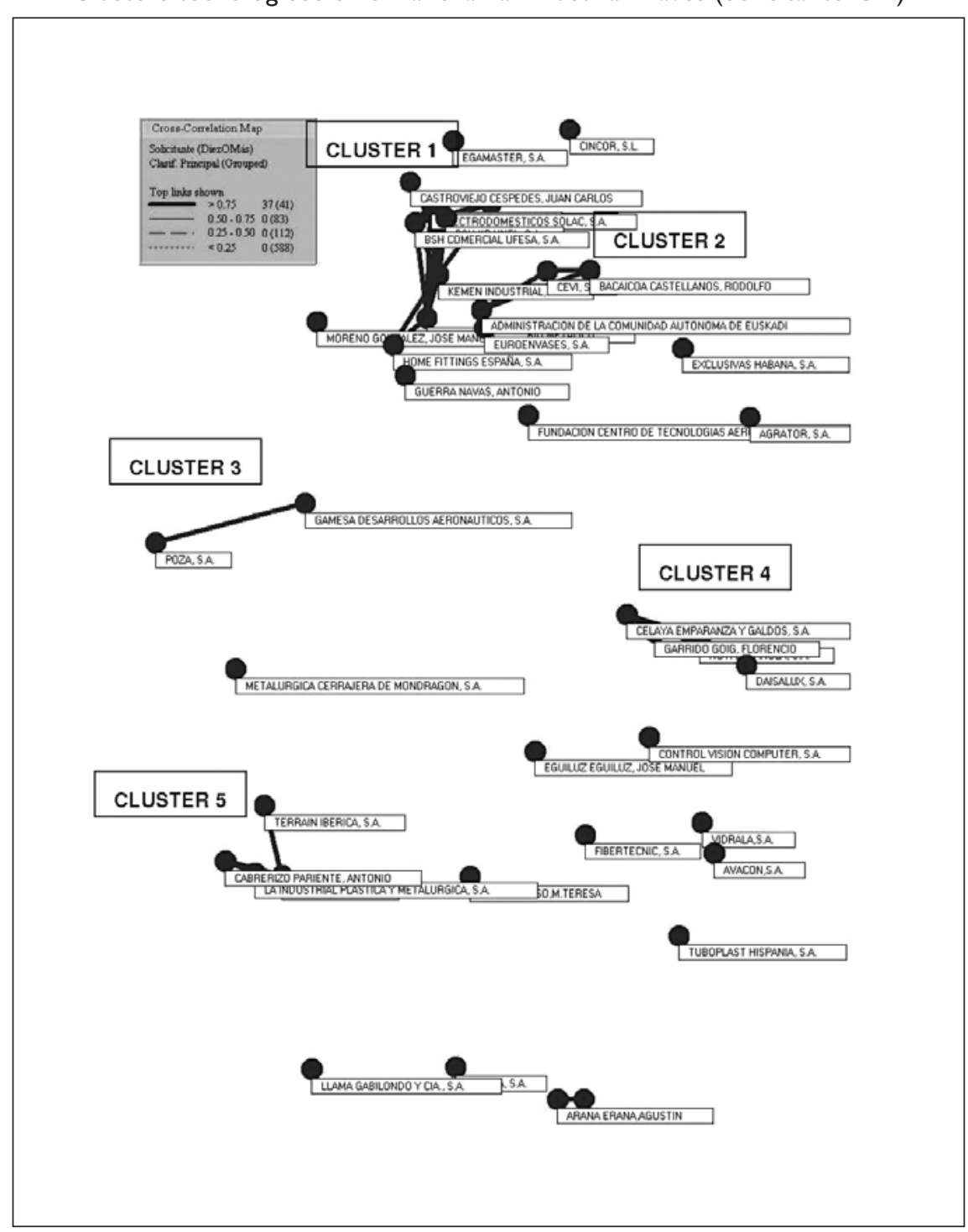

- CLUSTER 4: Celaya, Emparanza y Galdos S.A., Florencio Garrido Goig, Novi Española, Daisalux S.A..y otros. Este cuarto grupo de empresas tiene actividad innovadora en el sector de aparatos y maquinaria eléctrica y energía eléctrica.

- CLUSTER 5:Terrain Ibérica S.A., Terrain SDP,Antonio Cabrerizo Pariente, La Industrial Plástica y Metalúrgica S.A. y otros tienen en común innovar en el sector de elementos mecánicos.

\subsubsection{Nuevas áreas de patentabilidad. Áreas emergentes}

Como se puede observar en la Figura 6 que muestra las áreas emergentes de patentabilidad y sus relaciones, podemos detectar tres cluster alrededor de los cuales se esta configurando la innovación:

\section{CLUSTER |}

Sectores de: telecomunicaciones, tecnología audiovisual, tecnología médica y óptica. Estos sectores han desarrollado su actividad innovadora, principalmente, en la década de los noventa.

\section{CLUSTER 2}

A partir del 2000 destacan la ingeniería nuclear, los semiconductores y los productos farmacéuticos y 
Figura 6

Áreas emergentes de patentabilidad y sus relaciones (CIP-fechas solicitud)

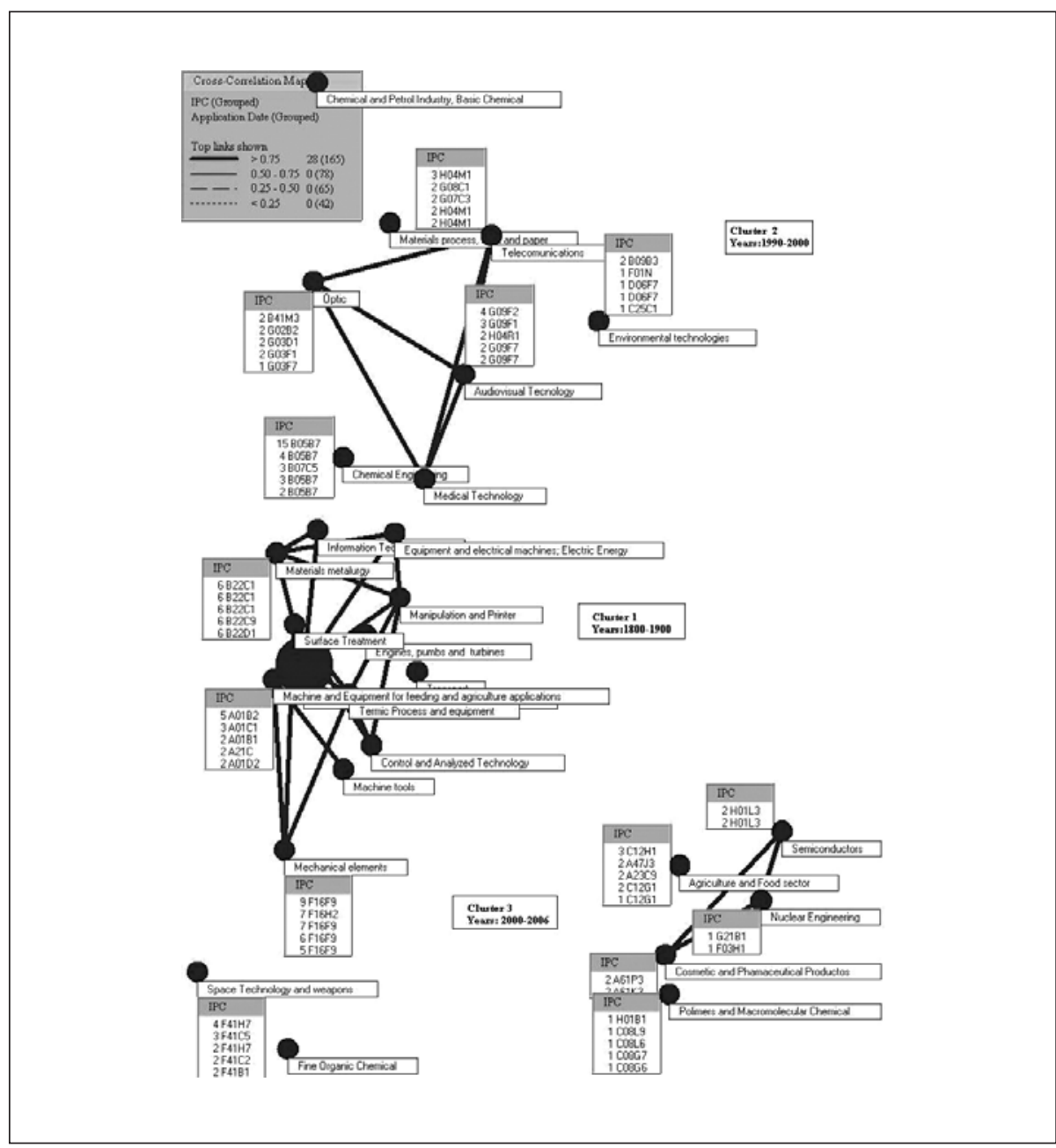

cosméticos. Cada uno de estos sectores sólo cuenta con una patente, solicitada en el intervalo del año 2000 al 2003, son por tanto, sectores emergentes innovadores.

\section{CLUSTER 3}

Integrado por los siguientes sectores: tecnologías de la información, materiales, metalurgia, aparatos y maquinaria eléctrica; energía eléctrica, ingeniería civil, construcción y minería, manipulación, impresión, maquinaria y aparatos para alimentación y agrícola, bienes de consumo y equipos, tratamientos superficiales, recubrimientos, tecnología de control, análisis y medida, procesos térmicos y aparatos, máquinas herramienta, elementos mecánicos y motores, bombas y turbinas. Dentro de estos sectores industriales, el mayor número de patentes se han producido en las décadas de los años 80 y 90 .

\section{Avance de conclusiones. Tendencias}

- La industria alavesa innova y lo refleja en la solicitud de Patentes de invención y Modelos de Utilidad. Podemos determinar que los sectores industriales que han movilizado la I+D en Alava han sido con un $37,28 \%$ la ingeniería civil y los bienes de consumo, en un 25,72\% la ingeniería mecánica y maquinaria, en un 18,93\% la ingeniería de proceso, el 8,67\% la electricidad y electrónica el 8, I 4\% la instrumentación, y con el I,26\% la Química-Farmacia.

- Las patentes solicitadas son bastante robustas ya que su rechazo en la oficina española de patentes y marcas es escaso solo un $8 \%$. Cuando se llega a registrar la invención es de gran calidad y novedad mundial.

- Analizando la evolución de los sectores que han patentado desde 1960 hasta la actualidad se apre- 
cia un cambio en lo que podíamos denominar «usabilidad» de la patente, es decir, se ha producido un cambio en las áreas de la innovación desde patentes de procesos industriales que daban lugar a productos finales hasta otras áreas donde se patentan mejoras de productos intermedios que se incorporan en el proceso productivo, por ejemplo, la llave alavesa (innovación de la denominada llave inglesa)

- Solo el 3\% de las patentes solicitadas son extendidas por vía internacional (PCT y Europea) principalmente se extienden a Europa en un 55\%.

- También se aprecia una disminución de la solicitud de patentes desde el año 1979 hasta el 2006. Podemos constatar que las medidas de fomento de la I+D no han sido suficientes, los fondos públicos se han dirigido a evitar la perdida de puestos de trabajo pero no se ha impulsado la generación de conocimiento y de actividades innovadoras. El resultado es una tendencia decreciente en la capacidad de I + D de la región. Señalar que la empresa con más patentes solicitadas de Alava y 65 de ellas en vigor denominada Inovac- Rima S.A. cerró sus puertas en el año 2005.

- Los polígonos industriales maduran y languidece su capacidad innovadora. En los últimos años, a partir del 2000, es el Parque tecnológico el que aglutina las empresas más innovadoras de la región.

- Al 29\% de las patentes solicitadas y concedidas le quedan más de diez años de vida, y corresponden a sectores como semiconductores, telecomunicaciones, productos farmacéuticos y cosméticos, tecnología médica y óptica. Ellos representan el motor alavés.

\section{Agradecimientos}

Se agradece a la Ingeniera en Organización Industrial Dña. Eva Gloria Sánchez Rexach su apoyo en el tratamiento de la información para la realización de este trabajo.

\section{Referencias}

DOU, H. H.-M. (2004). Benchmarking R\&D and companies through patent analysis using free databases and special software: a tool to improve innovative thinking. World Patent Information. Vol. 26, pp. 297-309

HAN, Y. J., and PARK, Y. (2006). Patent network analysis of inter-industrial knowledge flows: the case of Korea between traditional and emerging industries. World Patent Information. Vol. 28, pp. 235-247

FABRY, B.; HOLGER, E.; LANGHOLZ, J., and KÖSTER, M. (2006). Patent portfolio analysis as a useful tool for identifying R\&D and business opportunitiesan empirical application in the nutrition and health industry. World Patent Information.Vol. 28, pp. 2I5-225

GRAY, P. H., and MEISTER, B. (2006). Knowledge sourcing methods. Information\& management. Vol 43, pp. $142-156$

HIDALGO,A. (2003). Los Patrones de Innovación en España a través del análisis de Patentes. Un análisis cualitativo en el período 1988-1998. Ed. Ministerio de Ciencia y Tecnología. Madrid.

TANSEY, M., and STEMBRIDGE, B. (2005). The challenge of sustaining the research and innovation process. World patent information. Vol. 27, pp. 21 2-226.

ZHU, D., and PORTER, A. L. (2002). Automated extraction and visualization of information for technological intelligence and forecasting. Technological forecasting and social change. Vol. 69, pp. 495-506. 